\title{
Desarrollo y validación del instrumento MEDUC-RX32, para la evaluación de docentes de programas de la especialidad de postítulo en radiología
}

\begin{abstract}
Alvaro Huete $G^{(1)}$, Rodrigo Julio $G^{(2)}$, Viviana Rojas $D^{(3)}$, Cristián Herrera $R^{(4)}$, Oslando Padilla $P^{(5)}$, Nancy Solís $L^{(6)}$, Margarita Pizarro $R^{(6)}$, Lorena Etcheberry $R^{(7)}$, Alberto Sarfatis $F^{(8)}$, Gonzalo Pérez $D^{(8)}$, Alejandro Delfino $Y^{(9)}$, Estrella Muñoz $V^{(3)}$, Horacio Rivera $B^{(3)}$, Marcela Bitrán $C^{(10)}$, Arnoldo Riquelme $P^{(11)}$.
\end{abstract}

1. Médico. Departamento de Radiología. Pontificia Universidad Católica de Chile. Santiago de Chile.

2. Médico del Servicio de Medicina Interna, Hospital Sótero del Río. Santiago de Chile.

3. Psicólogo. Pontificia Universidad Católica de Chile. Santiago de Chile.

4. Médico. Salud Pública. Pontificia Universidad Católica de Chile. Santiago de Chile.

5. Estadistico. Pontificia Universidad Católica de Chile. Santiago de Chile.

6. Bioquímico. Pontificia Universidad Católica de Chile. Santiago de Chile.

7. Sociologo. Pontificia Universidad Católica de Chile. Santiago de Chile.

8. Medicina Interna. Pontificia Universidad Católica de Chile. Santiago de Chile.

9. Anestesiólogo. Pontificia Universidad Católica de Chile. Santiago de Chile.

10. Centro de Educación Médica. Pontificia Universidad Católica de Chile. Santiago de Chile.

11. Departamento de Gastroenterología. Pontificia Universidad Católica de Chile. Santiago de Chile.

Development and validation of the MEDUC-RX32 questionnaire, to evaluate teachers of postgraduate radiology programs

Abstract: The objective of this study is to develop and validate a guideline for the evaluation of the clinical teaching process in radiology; Catholic University Medical School, Radiology 32 items (MEDUC-RX32). A mixed methodology was used for the development of the questionnaire; two interview groups (residents and faculty) and one individual to a radiology program head. Using a modified Delphi technique to reach an agreement, a national validity panel assessed the importance of each item. The Delphi panel refined the questionnaire from 88 to 32 items after two rounds. The final guideline was perfomed in 55 residents of the program. The final instrument shows high reliability (Cronbach's alpha coefficient of 0.957). The average performance evaluations of teachers was $6.23 \pm 0.8$ (Likert scale 0 to 7) becoming a valid and reliable guideline for teacher evaluation of programs in the specialty of radiology, in Spanish speaking countries. Keywords: Assessment guidelines, Medical education, Postgraduate, Radiology teacher evaluation.

Resumen: El objetivo de este estudio es desarrollar y validar una pauta para la evaluación del proceso de enseñanza clínica en radiología; Medicina Universidad Católica, Radiología 32 ítems (MEDUC-RX32). Se utilizó metodología mixta para el desarrollo del instrumento: dos entrevistas grupales (residentes y docentes) y una individual a un jefe de programa de radiología. Utilizando técnica Delfi modificada para lograr acuerdo, un panel de validez nacional evaluó la importancia de cada ítem. El panel Delfi refinó el instrumento de 88 a 32 ítems luego de dos rondas. La pauta final fue piloteada en 55 residentes del programa. El instrumento definitivo presenta alta confiabilidad (coeficiente alfa de Cronbach de 0,957). El promedio de las evaluaciones del desempeño de los docentes fue de 6,23 $\pm 0,8$ (escala Likert 0 a 7) constituyéndose en una pauta válida y confiable para la evaluación de docentes de programas de la especialidad de radiología en países de habla hispana.

Palabras clave: Educación médica, Evaluación docentes radiología, Pautas de evaluación, Posgrado.

Proyecto de investigación financiado por la Dirección de Postgrado de la Pontificia Universidad Católica de Chile y por el Fondo Nacional de Desarrollo Científico y Tecnológico (FONDECYT) proyectos No 1120652 (A.R.) y No 1120534 (M.B.)

Huete $A$, et al. Desarrollo y validación del instrumento MEDUC-RX32, para la evaluación de docentes de programas de la especialidad de postítulo en radiología. Rev Chil Radiol 2014: 20(2): 75-80.

Correspondencia: Arnoldo Riquelme, MMedEd / a.riquelme.perez@gmail.com

Trabajo recibido el 15 de marzo de 2014. Aceptado para publicación el 22 de mayo de 2014. 


\section{Introducción}

Los programas de postgrado en medicina han tenido un gran desarrollo en los últimos años, con el advenimiento de nuevas tecnologías y la necesidad de mayor conocimiento en el diagnóstico y manejo específico de enfermedades complejas ${ }^{(1)}$. El desarrollo de estos programas se basa en la capacidad de transmisión de conocimientos, destrezas y actitudes de parte de especialistas (docentes) altamente calificados. Las fortalezas de dichos docentes incluyen sus habilidades en el proceso de diagnóstico basado en imágenes y exámenes de laboratorio así como en el manejo de pacientes, pero que -por lo generalno tienen una formación profesional en los aspectos relacionados con la docencia ${ }^{(2)}$.

La mayoría de las habilidades del docente clínico se vinculan a la transferencia de destrezas clínicas a los residentes, control de sesiones de pequeño grupo (seminarios) y reuniones clínicas, rol de facilitador o de modelo clínico, entrega de retroalimentación (feedback) y evaluación ${ }^{(3)}$. Sin embargo, otras áreas del quehacer docente que se relacionan con inter-profesionalismo, habilidades comunicacionales, medicina basada en la evidencia, mentoría y acompañamiento de residentes en problemas, constituyen habilidades del educador médico de postgrado que emergen con una creciente relevancia en los últimos años ${ }^{(4)}$.

Otros roles del educador médico se relacionan con el diseño curricular, identificación y desarrollo de competencias médicas o la conducción de procesos de acreditación de programas, pero éstos son habitualmente ignorados o poco desarrollados, ya que se asocian a un organizador de cursos más que a un docente clínico ${ }^{(5)}$. Más aún, el nuevo perfil del educador médico de postgrado debería incluir desafíos relacionados con nuevos conceptos e innovación en educación médica, los que incluyen el desarrollo curricular de programas de acuerdo a competencias ${ }^{(6)}$, uso de metodologías de enseñanza que promuevan el aprendizaje independiente como el portafolio ${ }^{(7)}$, uso de simulaciones para el aprendizaje de procedimientos prácticos ${ }^{(8)}$, enseñanza a distancia, entrega de retroalimentación efectiva, uso de instrumentos de evaluación acordes a lo que se desea calificar, como preguntas de selección múltiple o ensayos (conocimiento) y de evaluación de destrezas clínicas, mediante exámenes objetivos estructurados (ECOE) o de destrezas en el sitio de trabajo ${ }^{(9)}$.

La evaluación de docentes clínicos por parte de los alumnos, cuenta con varios instrumentos que incluyen diversos aspectos relacionados con las habilidades previamente descritas ${ }^{(10-15)}$ entre los que destacan: el Maastricht Clinical Teaching Questionnaire (MCTQ) $)^{(10)}$, que es considerado el cuestionario con mayor rigurosidad metodológica en su desarrollo y validación psicométrica, orientado a la docencia hospitalaria, el instrumento Medicina Universidad Católica - 30 ítems
(MEDUC-30) ${ }^{(15)}$, creado en la Facultad de Medicina de la Pontificia Universidad Católica de Chile (FMPUC) por Bitrán y colaboradores para la evaluación de profesores clínicos de pregrado y otros desarrollados en el extranjero como el Student Evaluation of Teaching in Outpatient Clinics (SETOC) ${ }^{(14)}$, el cuestionario Stanford Faculty Development Program (SFDP26) ${ }^{(12)}$ y la University of Michigan Global Rating Scale (GRS) ${ }^{(13)}$ enfocados en la formación de postgrado ambulatorio.

Existen experiencias de evaluación de la labor docente por parte de residentes en programas de formación con actividad centrada a nivel intrahospitalario(16-17), incluyendo el desarrollo de pautas de evaluación genéricas en el ámbito de la docencia en radiología ${ }^{(18)}$. Algunas de éstas han identificado conductas específicas asociadas a la buena docencia y demostrado que, a través de retroalimentación dirigida, los docentes con mayores falencias pueden progresivamente ir mejorando sus capacidades como tutores clínicos ${ }^{(18)}$. En la actualidad la docencia clínica tutorial en radiología ocurre fundamentalmente durante las sesiones de interpretación e informe de casos, así como también durante la realización de exámenes diagnósticos (ecotomografías, estudios fluoroscópicos) y procedimientos diagnósticos/ terapéuticos guiados por imagen. La interacción docente-residente durante las sesiones de informe supone la aplicación de múltiples estrategias de enseñanza y aprendizaje en el lugar de trabajo, con algunas diferencias relevantes en comparación a especialidades cuya labor clínica suponga un contacto directo con el paciente. Tomando en consideración estos elementos distintivos y frente a la falta de instrumentos validados para la evaluación de docentes por parte de los residentes de la especialidad, se creó un grupo de trabajo multidisciplinario que incluye radiólogos, residentes, especialistas en educación médica, bioquímicos vinculados a investigación en educación, psicólogos, sociólogos y estadísticos, cuyo objetivo es desarrollar y validar un instrumento de evaluación del desempeño de docentes clínicos de postgrado en Radiología; Medicina Universidad Católica - Radiología 32 ítems (MEDUC-RX32).

\section{Métodos}

El estudio consideró una metodología mixta, incluyendo una fase cualitativa y otra cuantitativa ${ }^{(19)}$. Para el componente cualitativo, se realizó una entrevista semiestructurada al director del programa de formación de postítulo en radiología de la FMPUC y dos entrevistas grupales focales a residentes y docentes. Los participantes del grupo focal de residentes, fueron invitados en forma personal considerando un muestreo aleatorio estratificado que garantizara la representatividad de los distintos niveles de avance en la formación, constituyéndose el grupo con 8 miembros. Por otro lado, los participantes del grupo 
focal de docentes clínicos, fueron invitados en forma personal atendiendo a un muestreo intencionado que asegurara la presencia de todas las subespecialidades y categorías académicas, quedando conformado también por 8 personas.

Se empleó análisis de contenido para llegar a conformar las categorías, apoyándose en elementos basados en la Teoría Fundada ${ }^{(20)}$. Se realizó una categorización abierta de los conceptos obtenidos y se empleó en el análisis la técnica informatizada (Software, Atlas ti). La información recogida se trianguló con la participación de dos analistas y se consideró saturada tras la revisión exhaustiva del material extraído de las tres fuentes mencionadas ${ }^{(19)}$. Los resultados fueron sometidos a los criterios de control de calidad propuestos por Ruiz(21).

Para el análisis cuantitativo, se realizó una técnica Delfi modificada con un panel de expertos quienes respondieron, en dos rondas, sobre el grado de importancia de los ítems, con una escala de Likert, donde: $0=\sin$ importancia, $1=$ poca importancia, $2=$ regular importancia, $3=$ importante y $4=$ muy importante. Luego de refinados los ítems, se construyó una encuesta piloto aplicada a residentes de radiología de la FMPUC, quienes evaluaron a sus docentes mediante una escala Likert de 0 a 7 , donde: $0=$ no aplica, $1=$ muy deficiente, $3=$ insuficiente, $4=$ suficiente, $5=$ bueno, $6=$ muy bueno y $7=$ excelente/sobresaliente. Posteriormente, se realizó análisis de confiabilidad del instrumento MEDUC-RX32, mediante el método de Cronbach alfa ( 0 a 1 ) en el que un valor mayor a 0,8 se considera de alta confiabilidad ${ }^{(22)}$.

El proyecto fue aprobado por el comité de ética de la FMPUC y todos los participantes firmaron consentimiento informado al participar en las entrevistas individual / grupal focal. Los resultados de las entrevistas individual / grupal focal fueron confidenciales y las encuestas fueron respondidas de manera anónima.

\section{Resultados}

Para la elaboración de los ítems, se consideraron las categorías identificadas a partir de la entrevista individual al jefe de programa de formación de postítulo en radiología de la FMPUC, los contenidos de ambos grupos focales y los aspectos identificados en otro instrumento de evaluación de docencia MEDUC30, creado en la FMPUC por Bitrán y colaboradores ${ }^{(15)}$ para la evaluación de profesores clínicos de pregrado.

De esta primera etapa emergieron 27 categorías de elementos relevantes para la docencia de la especialidad de radiología, que se expresaron en 88 ítems (Figura 1). En una segunda instancia, el grupo de investigadores realizó un proceso de refinamiento, obteniéndose 57 ítems, los cuales se ofrecieron a un panel de 34 expertos nacionales (radiólogos con cargo o actividad docente en programas de formación, tanto en la Región Metropolitana como de otras regiones del país), usando técnica Delfi modificada (escala Likert 0-4). En la tercera etapa del proyecto, el instrumento preliminar fue administrado en forma electrónica al panel de expertos. Los ítems con puntaje $>3$ se consideraron relevantes en ambas rondas Delfi y se eliminaron los que tuvieron un puntaje menor. La tasa de respuesta fue $91,2 \%$ y se obtuvieron 51 ítems en la primera ronda y 32 luego de la segunda ronda, como se ilustra en la figura 1. En la cuarta etapa se confeccionó un instrumento piloto (MEDUC-RX32), el cual se administró a 55 residentes de la especialidad.

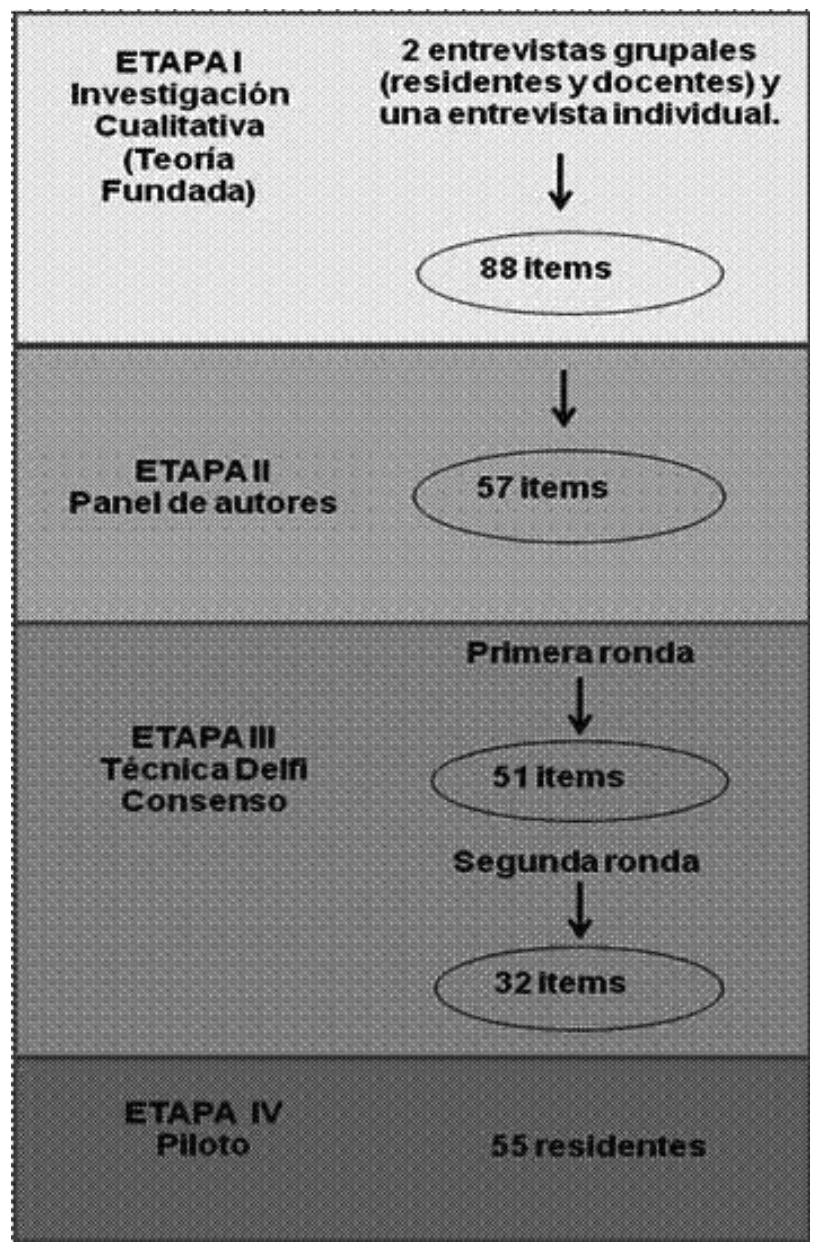

Figura 1. Diagrama de flujo del proceso de refinamiento de MEDUC-RX32.

El instrumento definitivo (Tabla I) presenta una alta confiabilidad (coeficiente alfa de Cronbach de 0,957). El promedio de las evaluaciones del desempeño de los 17 docentes fue de 6,23 $\pm 0,8$ (escala 0 a 7 ). Las fortalezas de los docentes se vincularon al trato respetuoso al residente y al equipo de salud (ítems $2,13,21,25$ y 27), trabajo en equipo (ítems 4 y 14) y razonamiento diagnóstico (ítem 22). Los aspectos detectados donde existe margen para mejorar incluyen entrega de retroalimentación (feedback) oportuno 
Tabla I. Resultado de la aplicación del instrumento piloto MEDUC-RX32

1. Él/ella me hizo preguntas que estimularon mi pensamiento crítico

2. Él/ella tuvo un trato deferente y respetuoso con todos los integrantes del equipo de salud

3. Él/ella planteó los problemas relacionados con el costo/ beneficio del uso de técnicas de imagen en las decisiones relativas al manejo de los pacientes

4. Él/ella informó oportunamente al equipo tratante en casos con hallazgos radiológicos de urgencia

$6,78 \pm 0,66$.

$6,38 \pm 0,88$

5. Él/ella recalcó la importancia de mis informes radiológicos como parte fundamental del proceso diagnóstico y terapéutico

6. Él/ella me demostró con su forma de trabajar que el radiólogo es parte importante del equipo tratante.

7. Él/ella colaboró con el manejo de la carga asistencial de la rotación en períodos de mayor demanda laboral.

8. Él/ella me comunicó mis avances y debilidades, sin esperar el fin de la rotación

9. Él/ella me ayudó a desarrollar gradualmente mi capacidad de tomar decisiones clínicas en forma autónoma.

10. Él/ella valoró mis preguntas y aportes como contribuciones al proceso de diagnóstico radiológico.

11. Él/ella me traspasó progresivamente la responsabilidad de redactar el informe radiológico

12. Él/ella me estimuló a actualizar mis conocimientos y prácticas por cuenta propia.

$6,62 \pm 0,70$

13. Él/ella mantuvo un comportamiento respetuoso con el médico solicitante del examen y sus hipótesis diagnósticas, aunque ellas fueran erradas.

$6,37 \pm 0,90$

$6,53 \pm 0,66$

$6,39 \pm 1,47$

$5,59 \pm 1,44$

$6,23 \pm 0,80$

$6,37 \pm 0,90$

$6,22 \pm 1,15$

$6,30 \pm 0,85$

$6,73 \pm 0,49$

14. Él/ella me enseñó que para realizar bien mi trabajo debo trabajar en equipo, (tecnólogos, enfermeros, técnicos paramédicos y administrativos)

15. Él/ella tuvo la habilidad de adecuar su docencia a la etapa de aprendizaje en la que me encuentro.

16. Al inicio de la rotación, él/ella me dio a conocer los objetivos de la rotación y las actividades docenteasistenciales correspondientes a mi nivel.

17. Él/ella me evaluó de acuerdo a los objetivos de la rotación, considerando el nivel que me corresponde.

18. Él/ella entregó indicaciones específicas para la realización de determinados exámenes

19. Él/ella me enseñó a redactar informes, indicando mis fortalezas y debilidades en su ejecución.

20. Él/ella creó situaciones que permitieran la exposición, discusión y seguimiento de casos generados en las actividades asistenciales.

21. Él/ella me respetó como persona y como médico.

22. Él/ella explicitó el razonamiento que le llevó a formular las hipótesis diagnósticas en base a las imágenes obtenidas durante el examen radiológico.

23. Él/ella me ayudó a resolver mis dudas y manejar situaciones especialmente complejas durante los turnos de residencia.

24. Él/ella asumió los errores propios y ajenos como oportunidades para el aprendizaje.

25. Él/ella mostró apertura a recibir preguntas y me enseñó en forma no intimidante.

26. Él/ella mostró interés por mi proceso de aprendizaje.

27. Él/ella me mostró cómo entregar, en forma breve, comprensible y respetuosa, información

al paciente sobre el examen o procedimiento que se le practicará.

28. Él/ella me alentó a interactuar con otros médicos y profesionales de la salud en la relación interdisciplinaria

29. Él/ella demostró entusiasmo por su labor docente, estimulándome a desarrollar mis habilidades docentes.

30. Él/ella me enseñó la importancia de presentar los casos radiológicos en forma clara y didáctica para diferentes audiencias.

$6,67 \pm 0,61$

$6,44 \pm 1,02$

$5,91 \pm 1,46$

$6,23 \pm 0,89$

$6,29 \pm 0,92$

$5,98 \pm 1,07$

$6,29 \pm 0,85$

$6,84 \pm 0,63$

$6,56 \pm 074$

$6,48 \pm 0,97$

$6,53 \pm 0,87$

$6,55 \pm 0,83$

$6,31 \pm 1,13$

$6,49 \pm 0,88$

$6,56 \pm 0,64$

$6,15 \pm 1,17$

$6,16 \pm 1,13$

31. Él/ella me orientó en el empleo de métodos apropiados de búsqueda de información para optimizar la interpretación radiológica de casos interesantes o complejos.

32. Él/ella me incorporó gradualmente en los procedimientos guiados por imagen que se incluyen en el programa de formación (observador-ayudante-efector supervisado). 
(ítem 8), métodos de búsqueda de información (ítem 31), enseñanza vinculada a la redacción de informes radiológicos (ítem 19) y entrenamiento en procedimientos guiados por imagen (ítem 32). En términos organizacionales, también existe una falencia en la comunicación de los objetivos de la rotación (ítem 16) que pudiera ser considerado dentro de los aspectos a mejorar.

\section{Discusión}

Si bien la adecuada organización de los aspectos administrativos y logísticos que rodean la implementación de todo programa de formación de especialistas médicos es una medida de gran relevancia para promover el éxito del aprendizaje de los residentes, ninguna es más efectiva que disponer de un grupo de docentes altamente motivados y con cualidades personales y pedagógicas apropiadas para desarrollar la labor del tutor clínico ${ }^{(2,17)}$.

La mayoría de los médicos que se desempeñan como docentes clínicos entrenando a residentes tienen excelentes credenciales como especialistas, con aptitudes y actitudes orientadas al correcto estudio y manejo de determinados pacientes / patologías. Sin embargo, salvo escasas excepciones, no han recibido entrenamiento formal ni capacitación en el desarrollo de sus competencias como docente ${ }^{(2)}$.

Tal como ocurre en la formación de residentes, una de las herramientas más poderosas para enfatizar conductas apropiadas y/o modificar debilidades en la labor clínica tutorial es la aplicación de retroalimentación o feedback ${ }^{(3,17)}$. Cuando ésta es específica (referida a conductas o desempeños determinados) y oportuna se convierte en una estrategia de gran utilidad en la evaluación docente. Esta retroalimentación puede basarse en pautas de cotejo que contengan indicadores relevantes al rol del docente ${ }^{(17)}$. En el caso de radiología, la enseñanza tutorial ocurre en gran medida en las sesiones de interpretación e informe de exámenes imagenológicos, durante las cuales no sólo se está realizando una actividad asistencial, sino que además se utiliza este escenario como sesión docente situada en el lugar de trabajo. Con frecuencia están presentes alumnos o internos de medicina (con conocimientos basales y objetivos docentes distintos de los residentes de especialidad), residentes de distintos niveles de formación y residentes de otras especialidades. Es así como se genera una sesión de aprendizaje en grupo pequeño, debiendo el radiólogo aportar no sólo sus conocimientos como experto médico, sino que demostrar un apropiado modelaje, capacidad de control de sesión, nivelar la enseñanza/retroalimentación de acuerdo a las necesidades particulares de los educandos y facilitar el estudio independiente, entre otras capacidades docentes $^{(4)}$. Las características docentes más relevantes, observables y evaluables por los residentes pueden ser resumidas como indicadores en una pauta de evaluación a ser llenada en forma periódica por los estudiantes.

La evaluación de los docentes del programa de radiología tuvo un buen alcance y permitió realizar una valoración de las fortalezas y de aspectos por mejorar de cada uno de los docentes evaluados por parte de los residentes. El instrumento demostró una alta confiabilidad que se encuentra en la línea del MEDUC30, previamente validado en pregrado de la FMPUC $^{(15)}$. La siguiente etapa del proyecto considera la aplicación a gran escala del MEDUC-RX32 para análisis de las propiedades psicométricas del instrumento incluyendo validez de constructo (análisis factorial) y estudio complementario de confiabilidad mediante teoría de generalización ${ }^{(23-24)}$.

En términos generales, la evaluación de la calidad de los docentes de radiología es más positiva que negativa, con espacio para mejorar.

Entre los aspectos positivos de los docentes de radiología, percibidos por los residentes al final de la rotación, se encuentran el trato respetuoso a los residentes, a otros integrantes del equipo de salud y al paciente, lo que se encuentra en sintonía con las fortalezas vinculadas al trabajo en equipos multidisciplinarios.

Por otro lado, entre los aspectos por mejorar destacan la falta de feedback oportuno, la falta de revisión sistemática de objetivos específicos con los residentes al inicio de cada rotación, el acompañamiento insuficiente en el proceso de redacción de informes y búsqueda de información para resolver problemas relacionados al proceso diagnóstico de pacientes, determinando algunos focos claros de intervención o capacitación docente a futuro.

Según Hutchinson, el desarrollo curricular de cursos y programas, así como la capacitación docente, son aspectos críticos vinculados directamente con la percepción de la calidad docente ${ }^{(25)}$. Entre las destrezas docentes susceptibles de capacitación destacan la organización de contenidos, enseñanza en aula o seminarios, el papel de supervisor y facilitador, destrezas propias de la profesión o especialidad, manejo de la información, habilidades comunicacionales, manejo de tecnología y evaluación ${ }^{(26)}$. La escuela de medicina donde tuvo lugar el presente estudio, se encuentra en constante revisión y actualización de su currículo ${ }^{(27-28)}$, incluyendo instancias de capacitación docente e implementación de iniciativas de innovación en educación médica en pre y postgrado(29).

Estos logros son en gran medida el reflejo de la capacitación de los docentes de pregrado ${ }^{(30)}$. Sin embargo, este apoyo sistemático no ha sido igual para los jefes de programa de postgrado ni para los docentes clínicos de residentes, quienes requieren habilidades diferentes a los tutores clínicos de pregrado(31).

En conclusión, el MEDUC-RX32 es un instrumento válido y confiable para la evaluación de docentes 
de programas de la especialidad de radiología en países de habla hispana. Este instrumento identifica las principales fortalezas y aspectos por mejorar de los docentes clínicos en esta especialidad médica y permite reconocer las áreas a abordar para mejorar la calidad de la enseñanza mediante capacitación docente. El cuestionario MEDUC-RX32, puede ser de utilidad en proceso de mejoramiento de calidad al interior de un programa de radiología y para el crecimiento profesional de cada uno de los docentes pertenecientes a dicho programa.

\section{Bibliografía}

1. Robin BR, McNeil SG, Cook DA, Agarwal KL, Singhal GR. Preparing for the Changing Role of Instructional Technologies in Medical Education. Acad Med 2011; 86: 435-439.

2. Davis DA, Prescott J, Fordis CM Jr., Greenberg SB, Dewey CM, Brigham T, et al. Rethinking CME: an imperative for academic medicine and faculty development. Acad Med 2011; 86: 468-473.

3. Holmboe ES, Ward DS, Reznick RK, Katsufrakis PJ, Leslie KM, et al. Faculty development in assessment: the missing link in competency-based medical education. Acad Med 2011; 86: 460-467.

4. Hesketh EA, Bagnall G, Buckley EG, Friedman M, Goodall $E$ et al. A framework for developing excellence as a clinical educator. Medical Education 2001; 35: 555-564.

5. Amin Z, Eng KH, Gwee M, Hoon TC, Rhoon KD. Addressing the needs and priorities of medical teachers through a collaborative intensive faculty development programme. Medical teacher 2006; 28: 85-88.

6. Cumming A, Ross M. The Tuning Project for Medicine-learning outcomes for undergraduate medical education in Europe. Med Teach 2007; 29: 636-641.

7. Riquelme $A$, Méndez $B$, de la Fuente $P$, Padilla $O$, Benaglio $C$ et al. Development and validation of a questionnaire on perception of portfolio by undergraduate medicalstudents. Rev Med Chil 2011; 139: 45-53.

8. Andresen M, Riquelme A, Hasbún P, Díaz C, Montana $R$, Regueira T. Evaluation of competencies for tracheal intubation among medical students. Rev Med Chil 2011; 139: 165-170.

9. Trivino X, Vásquez A, Mena A, López A, Aldunate M, Varas M, et al. Objetive Structured Clinical Examination for a pediatric internship assessment in two schools of medicine. Rev Med Chil 2002; 130: 817-824.

10. Stalmeijer RE, Dolmans DH, Wolfhagen IH, Muijtjens AM, Scherpbier AJ. The Maastricht Clinical Teaching Questionnaire (MCTQ) as a valid and reliable instrument for the evaluation of clinical teachers. Acad Med 2010; 85: 1732-1738.

11. Skeff KM, Stratos GA, Berman J, Bergen MR. Improving clinical teaching. Evaluation of a national dissemination program. Arch Intern Med 1992; 1 52: 1156-1161.

12. Litzelman DK, Stratos GA, Marriott DJ, Skeff KM. Factorial validation of a widely disseminated educational framework for evaluating clinical teachers. Acad Med
1998; 73: 688-695.

13. Williams BC, Litzelman DK, Babbott SF, Lubitz RM, Hofer TP. Validation of a global measure of faculty's clinical teaching performance. Acad Med 2002; 77: 1 77-180.

14. Zuberi RW, Bordage G, Norman GR. Validation of the SETOC instrument - Student evaluation of teaching in outpatient clinics. Adv Health Sci Educ Theory Pract 2007; 12: 55-69.

15. Bitran M, Mena B, Riquelme A, Padilla O, Sánchez I, Moreno R. An instrument in Spanish to evaluate the performance of clinical teachers by students. Rev Med Chil 2010; 138: 685-693.

16. Maker VK, Curtis KD, Donnelly MB. Faculty evaluations: diagnostic and therapeutic. Curr Surg 2004; 61: 597-601.

17. Baker K. Clinical teaching improves with resident evaluation and feedback. Anesthesiology 2010; 113: 693-703.

18. Cohan RH, Dunnick NR, Blane CE, Fitzgerald JT. Improvement of faculty teaching performance: efficacy of resident evaluations. Acad Radiol 1996; 3: 63-67.

19. O'Cathain A, Murphy E, Nicholl J. Three techniques for integrating data in mixed methods studies. BMJ 2010; 341: c4587.

20. Corbin J, Strauss A. Grounded Theory Research Procedures, Canons and Evaluative Criteria. Zeitschrift Fur Soziologie 1990; 19: 418-427.

21. Ruiz J. Metodología de la investigación cualitativa. Bilbao: Universidad de Deusto. Cap. 3: Control de calidad 1996.

22. Cronbach LJ. Coefficient alpha and the internal structure of tests. Psychometrika 1951; 16: 297-334.

23. Downing SM. Validity: on meaningful interpretation of assessment data. Med Educ 2003; 37: 830-837.

24. Boor K, Scheele F, Van der Vleuten CP, Scherpbier AJ, Teunissen PW, Sijtsma K. Psychometric properties of an instrument to measure the clinical learning environment. Med Educ 2007; 41: 92-99.

25. Hutchinson L. Educational environment. BMJ 2003; 326: 810-812.

26. Castillo M. The teaching profession. Rev Med Chil 2010; 138: 902-907.

27. Rosso P, Velasco N, Moreno R. Undergraduate curriculum reform at the Pontifical Catholic University Medical School: aims, methodology and advance status. Rev Med Chil 1997; 125: 796-807.

28. Bitran M, Wright AC, Zuñiga D, Mena B, Velasco N, Moreno R. Improvement of medical student's academic performances in times of curricular reform. Rev Med Chil 2002; 130: 437-445.

29. Sánchez I, Riquelme A, Moreno R, Mena B, Dagnino J, Grebe G. Revitalising medical education: the School of Medicine at the Pontificia Universidad Católica de Chile. The Clinical Teacher 2008; 5: 1-5.

30. Trivino X, Sirhan M, Moore P, Montero L. Impact of a diploma on medical education in a medical school in Chile. Rev Med Chil 2011; 139: 1508-1515.

31. Herrera C, Niklitschek I, Pizarro M, Solís N, Olivos T, Rojas $\mathrm{V}$, et al. Identifying the main training needs of postgraduate medical program managers. Rev Med Chil 2013; 141: 1126-1135. 